ISSN: $1130-3743$

\title{
REFLEXIÓN Y COLABORACIÓN EN LOS ENTORNOS EDUCATIVOS: HACIA LA PROFESIONALIZACIÓN DOCENTE
}

\section{REFLECTION AND COLLABORATION IN THE EDUCATIONAL CONTEXT: TOWARDS PROFESSIONAL TEACHING}

\author{
JUANA MARÍA RODRÍGUEZ GÓMEZ \\ Universidad de La Laguna. Centro Superior de Educación. C/. Heraclio Sánchez, no 37. \\ 38204 La Laguna.
}

\section{RESUMEN}

El profesor como crítico y reflexivo de su acción, ha de participar en la creación del conocimiento pedagógico y en la toma de decisiones de los procesos educativos.

Las primeras experiencias en el aula constituyen un período clave en el desarrollo profesional del profesor. Conscientes de este tema, abordamos algunas líneas de actuación centradas en el conocimiento de la clase y una nueva organización del centro educativo basada en la colaboración, que le permitan descubrir y solucionar, con sus compañeros de trabajo, los distintos conflictos que diariamente observan en las aulas, desde el análisis riguroso de la teoría y práctica educativa.

\section{SUMMARY}

The teacher as a critic and reflexive subject of his own action, has to participate in the creation of the pedagogic knowledge and in marking decisions to the educative processes.

The first experiences in the class are an important period in the professional development of the teacher. Taking into account this topic, we deal with some ways of action focused on the class knowledge and an new organization of the educative place based on cooperation. These ways of action allow the teacher and his collegues to find and resolve the different conflicts which emerge in the class every day. These conflicts are viewed from the severe analysis of the educative theroy and practice. 


\section{INTRODUCCIÓN}

Entendemos al profesor como un profesional reflexivo, comprometido con el entorno socio-cultural, que promueve una nueva concepción de la enseñanza y de la relación teoría-práctica educativa.

Las primeras experiencias en el aula representan un momento clave en el desarrollo profesional del profesor. Conscientes de este tema y de las numerosas dificultades encontradas, abogamos por un conocimiento adecuado de la clase y una organización del centro educativo basado en la colaboración entre los docentes y entre éstos y la universidad.

Esta apuesta debe impregnar los programas de formación del profesorado, esbozando un modelo de prácticas de enseñanza que combine la reflexión y el análisis con la participación e intervención en el aula.

\section{EL PROFESOR COMO UN INVESTIGADOR REFLEXIVO}

Sthenhouse (1984) defiende una cultura profesional autónoma, comprometida con la transformación social y una actitud investigadora sobre la práctica. Este proceso investigador, asociado a la figura del docente, se transforma en autoindagación reflexiva, lo que supone una reconceptualización de la enseñanza y de la práctica educativa.

Entendemos la práctica como un proceso de investigación donde el profesor se sumerge en el mundo del aula para comprenderlo de forma crítica y vital, analizando sus mensajes, cuestionando sus propias creencias y planteamientos, contrastando interpretaciones y participando en la reconstrucción permanente de la realidad escolar.

Los profesores serán mejores profesionales si son conscientes de su prácti$\mathrm{ca}$, si reflexionan sobre sus intervenciones, como intelectuales y artistas, que utilizan el conocimiento científico para comprender la situación del aula así como para diseñar y construir estrategias flexibles, adaptadas a cada contexto; una tarea profesional apoyada en el diseño abierto, la investigación diagnóstica, la experimentación creadora y la evaluación reflexiva.

Esta visión del docente como crítico y reflexivo redefine la relación entre teoría y práctica. El profesor ha de participar en la creación del conocimiento pedagógico y en la toma de decisiones de los procesos educativos. Al respecto, añade Imbernón (1994).

"La formación del profesorado no se apoya tanto en la adquisición de conocimiento teórico de la disciplina académica y rutinas didácticas, como en el desarrollo de capacidades de análisis, indagación, reflexión crítica y procesamiento de la información para el diseño de proyectos antes y durante la acción" (Imbernón, 1994, p. 96).

Asimismo, equilibra una variedad de intereses que se necesitan satisfacer en la clase mediante la negociación (Lampert, 1985). Propone soluciones a los con- 
flictos que emergen del aula, los acepta y trata de encontrar la mejor manera de afrontarlos.

"Los profesores hacen elecciones entre las alternativas para promover la igualdad, para construir el curriculum según los intereses de los niños o sobre las asignaturas, para fomentar la independencia o la creatividad. Estas elecciones tratan de ahondar los problemas de su práctica cotidiana" (Lampert, 1985).

Tomando como referencia el grado de interacción en el aula, podemos establecer los siguientes estilos o imágenes, asociadas a los docentes (Lampert, 1985):

a) La imagen del inbibido. El profesor carece de objetivos claros, asediado por otros agentes educativos. Presenta numerosos problemas para interaccionar en la clase. Se inhibe ante las inconsistencias mostradas por los alumnos, la administración, los padres, tratando de afrontar el conflicto de forma traumática.

b) La imagen de reproductor. El profesor como reproductor del conocimiento y las técnicas diseñadas por los expertos. Apoyado en los agentes educativos externos trata de encontrar fórmulas y técnicas que aclaren o eliminen sus conflictos. Tiene la responsabilidad de controlar y dirigir el aprendizaje de sus alumnos.

c) La imagen del biperactivo. El profesor decide cómo debe dar su clase. Toma decisiones rápidas y eficaces sobre el proceso para lograr los objetivos deseados por el sistema educativo. Adopta el rol de un mecánico de la enseñanza, con actuaciones impersonales y válidas para todos, obviando cualquier alternativa que pudiera resolver el conflicto.

d) La imagen del crítico. El profesor reconoce el conflicto y trata de asumirlo como algo intrínseco a las situaciones educativas y no como algo a eliminar. Según Lampert (1995), reconocer el conflicto y vivir con él puede interpretarse como una debilidad de las ciencias humanas frente a las físico-naturales. Sin embargo, hemos de entender la complejidad de los fenómenos sociales y las dificultades que conlleva aislar todas sus variables para estudiarlas experimentalmente.

Por tanto, entendemos al docente como un profesional de la educación que es capaz de trabajar y prosperar en situaciones adversas o contradictorias. Si tenemos como objetivo la profesionalización del profesor, su actuación no puede reducirse a una mecanización rutinaria o a la improvisación, sino que debe apostar por acciones planificadas en correspondencia con la teoría que los sustenta. Es decir, frente a la rutina, hay que favorecer la racionalidad en y del docente, que depende de su habilidad para integrar y aplicar adecuadamente conocimientos y teorías a las situaciones de la clase.

Cuando la práctica docente no es guiada de forma científica, se improvisa. Según Gimeno y Fernández (1980), los programas de formación del profesorado no pueden fundamentarse en la fragmentación disciplinar, sino en la integración temática para ofrecer una respuesta coherente en la práctica. Esta relación teoríapráctica constituye un objetivo de la profesionalidad docente. Si esto no ocurriese así, la formación teórica quedaría inoperante y las decisiones de intervención 
en la práctica carecerían de eficacia, mediatizadas por el "sentido común pedagógicon y por sus teorías implícitas.

\section{LAS PRIMERAS EXPERIENCIAS COMO DOCENTES. LAS PRÁCTICAS DE ENSEÑANZA}

Las prácticas de enseñanza constituyen un momento clave de su desarrollo profesional, caracterizado por la confluencia de sentimientos ambivalentes, inseguros y contradictorios. Los futuros docentes deben acomodarse a dos instituciones educativas, la universidad y la escuela, con demandas distintas. Generalmente, la vida escolar invita a la acomodación e imitación de rutinas así como a la negación de objetivos personales en favor de las expectativas y demandas del colegio, que chocan con las argumentaciones y presupuestos teóricos defendidos desde la universidad.

Esta disonancia universidad-colegio afecta a todos los agentes educativos, alumnos en prácticas de enseñanza, maestros en ejercicio y profesores universitarios, que debe apostar por la colaboración y el análisis conjunto, necesario para abordar problemas asociados a la educación como (Tabachnick, 1980):

- Problemas referidos al conocimiento. Tradicionalmente, se han considerado a las escuelas como lugares donde los alumnos adquieren el conocimiento y la información, a través de la observación y la exploración. El conocimiento se presenta amplio frente a la brevedad del tiempo escolar. Por tanto, ¿qué información tendrá mayor significado para los niños? o ¿por qué ese conocimiento y no otro es necesario? Las respuestas ofrecidas por los profesionales de la educación pueden referise tanto a conceptos y procesos asociados a una disciplina como a propósitos éticos y políticos, derivados del entorno socio-cultural que rodea al centro educativo.

- Problemas de acción. Generalmente, se han trasladado los problemas de conocimiento a los problemas de ejecución y de intuición, es decir, cómo hacer que algo funcione en clase.

"Debido a la presión para decidir y actuar en el momento de crisis, la mayoría de la acción en clase es intuitiva y no está claro su significado" (Tabachnick, 1980, p. 24).

Las presiones grupales e institucionales para resolver el problema de la enseñanza se reflejan en la ansiedad del docente, deseoso de controlar a sus alumnos. Esta se entiende como un problema de actuación, como una forma exhaustiva de llevar a cabo las tareas rutinarias.

"La enseñanza se interrumpe y se rompe la cadena de ideas para calmar a los niños y hacerles trabajar. Esto es necesario a menudo porque el trabajo no ha sido planeado para responder a las diferencias en los intereses o niveles de habilidad. Los alumnos en prácticas tratan de responder al aburrimiento de los niños y los fuerzan a estar atentos" (Tabachnick, 1980, p. 24). 
- Problemas de personalidad. Desde la universidad se anima a los alumnos a ser independientes, a desarrollar un estilo profesional, alejado de conductas y patrones irreflexivos. Esta opción personal, que invita al desarrollo profesional, ocupa un segundo lugar cuando reconocemos las "necesidades de supervivencia", asociadas a este momento formativo, mediatizadas por la obtención de una buena o mala nota o por la necesidad de encontrar trabajo.

“La enseñanza se interrumpe y se rompe la cadena de ideas para calmar a los niños y hacerles trabajar. A menudo esto es necesario porque el trabajo no ha sido planeado para responder a las diferencias de los alumnos. Los alumnos en prácticas tratan de responder al aburrimiento de los niños y los fuerzan a ser atentos (Tabachnick, 1980, p. 24).

Tales mensajes contradictorios sobre la autonomía del profesor y el desarrollo del estilo personal de enseñanza caracterizan a las escuelas y determinan la relación con la universidad. Por ejemplo:

"En la mayoría de las entrevistas, los alumnos en prácticas dicen que quieren ser diferentes al maestro del colegio. Sugieren que quieren desarrollar un curriculum más variado y libre por los alumnos. Estos compromisos son contradictorios puesto que la mayoría de los estudiantes fallan a la hora de crear tal ambiente cuando tienen la oportunidad. Sus intereses están supeditados a sus necesidades de supervivencia" (Tabachnick, 1980, p. 25).

Reconociendo estos problemas educativos y las dificultades de los futuros docentes, abordamos algunas líneas de actuación centradas en el conocimiento de la clase y en una nueva organización docente basada en la colaboración y el análisis de los conflictos existentes en el aula y en el centro; temas que tratamos a continuación.

\subsection{Conocimiento de la clase}

El futuro docente no sólo debe conocer la materia que imparte y la metodología que utiliza sino cuáles pueden ser las demandas de la clase para tomar decisiones acertadas.

Las relaciones establecidas por el profesor y el alumno dentro del aula son recíprocas y se caracterizan por la ambigüedad, la disparidad y la prontitud (Doyle, 1977). Las clases están llenas de alumnos distintos, numerosas actividades y sugerencias así como contratiempos que desbordan al profesor. Este carece de tiempo real para reflexionar sobre su actuación (Doyle, 1979). Al respecto, Kounin (1970) identificó una serie de habilidades desarrolladas por el docente, orientadas a las demandas del ambiente en la clase: Habilidades para manejar dos situaciones a la vez; habilidades que implican a todos los alumnos en cada actividad y habilidades para generar y cambiar tareas.

Asimismo, se ha demostrado que el interés de los alumnos en la clase se asocia con el tipo de actividad presentada por el docente y con el tiempo de la 
misma. Kounin y Doyle (1975) descubrieron que la implicación del alumno en clase dependía de la estructuración de las actividades. Para estos autores, el éxito del trabajo en el aula está en función de la complejidad de la actividad llevada a cabo, la inclinación de los estudiantes a implicarse en la misma y la habilidad para realizar las operaciones demandadas (Doyle, 1977). De igual forma, tanto las actividades seleccionadas por el profesor como su distribución en el tiempo y los recursos utilizados dependen del ambiente creado en el aula (Woods, 1977). Por ejemplo, cuando no hay voluntarios en clase, el profesor suele escoger a aquellos que normalmente intervienen y aportan la respuesta correcta. Esta forma de seleccionar a los alumnos para que respondan tiene como argumento la necesidad de mantener la dinámica de las actividades de la clase (Doyle, 1979).

El estudio de los efectos de la clase en los docentes plantea numerosos interrogantes sobre lo que éstos hacen y cómo piensan sobre su trabajo. La enseñanza debe ir más allá de prescripciones para convertirse en un proceso singular que persigue un mayor entendimiento de los estudiantes y del entorno socio-cultural.

\subsection{1. ¿Qué hacen los alumnos en prácticas? ¿Cuál es el conocimiento real del aula?}

Tratamos de identificar y descubrir qué hacen los alumnos en prácticas cuando están en clase y cuál es su conocimiento real del aula. A menudo, los alumnos en prácticas están inmersos en una enseñanza rutinaria y mecánica que reitera el valor de los procedimientos y la evaluación, preocupados por hacer cosas para que los niños reciban a tiempo la lección de forma ordenada y tranquila. Así, muchas de las tareas diseñadas giran en torno a la repetición y memorización automática.

Tabachnick (1980) indicó que las actividades más comunes, asociadas a los alumnos en prácticas, pueden agruparse en torno a los siguientes puntos:

a) Estructuración del día escolar. Este se divide en espacios de tiempo, cada uno representado por una materia distinta. Estos espacios fueron establecidos por el centro y raramente se modificaban a lo largo del curso escolar. Por ejemplo, "leer en el tiempo de lectura". De tal forma que, los alumnos en prácticas recurrían al orden cuando los alumnos no aceptaban la estructura propuesta. Añadimos la siguiente respuesta:

"Normalmente leen una historia de diez páginas. Podemos leer la mitad y hacer preguntas mientras se lee o después de la lectura, para ver si recuerdan lo que han leido" (Tabachnick, 1980, p. 13).

b) Los libros y los cuadernos de clase. Estos constituían el material empleado por la mayoría de los alumnos en prácticas. Recogemos la descripción de uno de ellos:

"A los niños se les requería que leyeran los folios y fueron evaluados al final de la unidad... Este es mi objetivo, antes de dejar la escuela, corregir todo el tra- 
bajo del día, si es posible. Desde que hay tres niveles hay tres series de libros de trabajo..." (Tabachnick, 1980, pp. 13-14).

Los alumnos en prácticas tendían a seguir las lecciones contenidas en estos materiales de forma rutinaria. La mayoría de las tareas se establecieron con anterioridad al período de prácticas, lo que limitó la creatividad y originalidad de los futuros docentes. En algunos casos, éstos podían formular otros procedimientos o seleccionar los materiales mientras cumplieran los objetivos establecidos por el centro. Sin embargo, se observó que, en aquellos ejemplos donde los alumnos en prácticas podían elegir temas y procedimientos, solían priorizar las actividades rutinarias.

c) Enfasis en el orden, en el control para mantener a los niños ocupados y callados, y en la evaluación.

"A los alumnos en prácticas se les observó apagar las gimnasia... Estas actividades han ocupado un lugar importante en las vidas de los futuros profesores. Hacer que los niños hagan su tarea en silencio y ordenadamente se convirtió en un fin en si mismo más que un medio para un propósito educativo" (Tabachnick, 1980, p. 15).

\subsubsection{Interacciones del alumno en prácticas}

a) Interacciones con los niños. Las interacciones con los niños suelen ser breves, impersonales y relacionadas con la tarea. La mayoría de los alumnos en prácticas centran sus esfuerzos en responder a preguntas cortas sobre la tarea, ofreciendo consejos y sugerencias. La propia estructura de la jornada escolar les impide mejorar el nivel de interacción con sus alumnos.

"En muchos casos, a los niños se les pedía que se movieran de la clase según asignaturas. Había muy poco tiempo libre en el día para que los alumnos en prácticas pudieran interaccionar con los niños sin la excusa de una lección". (Tabachnick, 1980, p. 16).

Asimismo, los futuros docentes asumen un rol pasivo en las interacciones con los maestros del colegio y si ésta existe se centra en consejos y recomendaciones que reproducen los objetivos existentes.

b) Interacciones con el maestro del centro. Fundamentalmente, a los maestros les preocupa aquellos puntos relacionados con la vida cotidiana del centro. Por ejemplo, el interés concedido a las visitas de padres o las técnicas para mantener el control de la clase.

"En casi todas las clases, a los estudiantes se les aconsejó no sonreír hasta llegar a Navidad. Había que empezar duro al principio para ganarse el respeto de los niños y después relajarse" (Tabachnick, 1980, p. 16).

En líneas generales, se evitan los conflictos con los maestros del colegio aún cuando el conflicto se presenta, en algunos puntos, inevitable. Así respondió un alumno en prácticas: 
"Si hay problemas en clase, los alumnos en prácticas no hablamos con los maestros porque tenemos miedo de ofenderles. Además hay que recordar que su recomendación de cómo se enseña es lo más importante y no hay que arriesgarse». (Tabachnick, 1980, p. 17).

Esto hace que los debates establecidos entre el futuro docente y el maestro del centro, se centren exclusivamente en la discusión de procedimientos y técnicas para llevar la clase: Cómo lo haces, cómo poder conseguir los materiales, etc. En estos debates, las tareas ocupan un lugar importante para medir el aprendizaje de los alumnos. La referencia constante a los procedimientos desplaza a un segundo plano los planteamientos éticos y políticos así como la capacidad de análisis y de reflexión del futuro profesor.

"La dependencia del alumno en prácticas y la presión institucional que señala un tiempo para desarrollar las tareas, ha eliminado la reflexión seria y el análisis... Muchos alumnos piensan en tratar mejor a los niños, pero las cargas diarias impiden desarrollar energías para mejorar lo que se hace.. (Tabachnick, 1980, p. 18).

c) Interacciones con la Universidad. Los últimos Planes de Estudio sobre formación del profesorado han apostado por un docente reflexivo, crítico y autónomo en su labor diaria, capaz de desarrollar su propio estilo de enseñanza.

Bajo estos presupuestos teóricos, se les exige a los futuros profesionales de la educación que adopten diferentes estilos de enseñanza para que descubran aquellas formas que mejor respondan a sus deseos personales y profesionales. Esta toma de decisiones por parte del futuro docente, debe emerger desde una relación triádica potenciada por el maestro del centro, el profesor de la Universidad y el alumno en prácticas. Una relación necesaria para clarificar los roles que cada uno debe desempeñar mediante el intercambio de experiencias y la reflexión conjunta.

Sin embargo, esta libertad para discutir y debatir los estilos de enseñanza se verá limitada por las férreas estructuras de un currículum excesivamente planificado por el colegio.

\subsection{Propuesta colaborativa. La formación centrada en centros}

Inserto en los parámetros ecológicos que revalorizan por un lado, las creencias, los pensamientos y conductas de los profesores y, por otro, las escuelas en tanto que instituciones, ha emergido un movimiento que pone su énfasis en el desarrollo profesional centrado en la escuela, el desarrollo de los docentes en íntima relación con el ambiente en el cual desempeñan su actividad educativa, la escuela.

Los principios sobre los que se fundamenta esta concepción del desarrollo docente quedan recogidos en los siguientes puntos: la escuela como lugar donde surgen y se pueden resolver la mayor parte de los problemas de la enseñanza; los profesores como elementos determinantes del éxito de la formación orientada a la 
escuela; sensibilidad hacia los contextos educativos cambiantes, dinámicos, reflexivos y participativos.

Por consiguiente, esta formación centrada en la escuela se caracteriza por la creación de relaciones personales constructivas así como por la implicación y participación de las instituciones oficiales que intervienen en la formación de los futuros profesores.

Tanto el plan de formación del profesorado esbozado por el Ministerio de Educación y Ciencia (MEC) como los principios explicitados en el Diseño Curricular Base (DCB), la formación en los centros o las referencias al desarrollo centrado en la escuela, revalorizan la idea de centro como lugar de cambio (Escudero, 1993). Según Imbernón (1994), la escuela constituye un lugar privilegiado para la planificación de actividades formativas que favorecen la autonomía docente.

Esto implica concebir al centro educativo como una organización, con una cultura determinada que la distingue de otras instituciones. En ella, la actitud del profesor representa la fuerza clave que da sentido tanto al desarrollo profesional como al desarrollo institucional.

Desde esta perspectiva colaborativa, no es suficiente la implicación esporádica del profesor en un grupo de reflexión sobre la práctica, sino que además éste ha de convertirse en un crítico-reflexivo de la institución en la que desempeña su trabajo. De esta forma, los centros educativos podrán acercarse a lo que Kemmis (1988) denomina "comunidades críticas e interpretativas", que superan el carácter exclusivamente técnico, intuitivo y aislado de los centros educativos. Al respecto, añade Escudero (1993):

"El reconocimiento explícito de la construcción social del ser y deber ser de las escuelas y las consecuencias derivadas de este supuesto comporta la creación de un espacio participativo y colaborativo para el desarrollo escolar". (Escudero, 1993, p. 239).

Por consiguiente, el desarrollo basado en la escuela no se configura bajo un programa de actuación elaborado por parte de los "externos" (investigadores o gestores) sino como un proceso colaborativo y de autorrevisión que asume todo su protagonismo.

El desarrollo colaborativo en la escuela aspira a mejorar y modificar la educación, potenciando la capacidad de los profesores para elaborar un diseño crítico y reflexivo sobre su práctica. Esto conlleva adoptar una ideología y una práctica congruente, según la cual el control, el desarrollo de todo el proceso de autorrenovación reside en la propia escuela. Esto supera conceptual y metodológicamente la idea de intervención "sobre" la escuela y los profesores (Lieberman, 1989).

Una escuela colaborativa es una escuela comprometida con la interdependencia, la apertura, la comunicación, la autorregulación, la colaboración y la autonomía. Estos principios han de impregnar los procesos de mejora y de perfeccionamiento de los docentes, mediante tareas o actividades de autorrevisión, 
resolución de problemas, búsqueda de alternativas, desarrollo y evaluación colaborativa. Esta apuesta por lo colaborativo, reflejada en la planificación mutua, la observación, la formación recíproca, incide en el proceso enseñanza-aprendizaje, reforzando la idiosincrasia de lo organizativo y lo pedagógico.

La formación centrada en los centros educativos ofrece una oportunidad para superar las barreras conceptuales y estructurales de la formación práctica de los docentes. Frente al viejo modelo basado en la enseñanza entendida como ciencia aplicada, estas escuelas de desarrollo profesional o "escuelas colaborativas" (Zeichner, 1992) abogan por la investigación y la reflexión. Este autor explicita:

"Que los futuros docentes vean y valoren el desarrollo colaborativo del profesor y tengan oportunidades para participar en el proceso de cambio de las escuelas". (Zeichner, 1992, p. 118).

Desde el punto de vista de la formación docente implica analizar y valorar el aprendizaje y el programa curricular del futuro maestro desde un proyecto conjunto, reforzado por los profesionales de la escuela y los tutores universitarios.

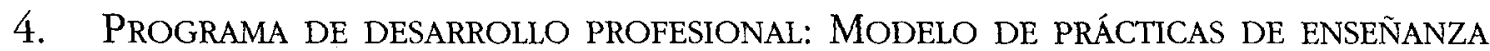

Según Zeichner y Liston (1987) los programas tradicionales de formación del profesorado han seguido un modelo de prácticas de enseñanza que ha inhibido el crecimiento directo de los futuros profesionales de la educación. Como contrapartida a esta situación desesperada, estos autores proponen un programa centrado en la reflexión y autonomía docente, bajo los parámetros de la participación crítica y democrática.

Tomando como referencia la "acción reflexiva" desarrollada por Dewey, se pretende que los alumnos en prácticas o futuros docentes asuman valores como la tolerancia y la responsabilidad en sus acciones. Un profesor reflexivo es aquel que evalúa los propósitos y consecuencias de su trabajo desde criterios educativos y morales. Por tanto, los alumnos en prácticas deben ser conscientes de su labor social y educativa para definir objetivos en el campo moral, educativo e instrumental.

Este programa de desarrollo profesional extiende sus raices en los siguientes principios: Preparación del profesor para reflexionar sobre los orígenes, propósitos y consecuencias de sus acciones; desarrollo de hábitos pedagógicos y de habilidades necesarias para dirigir el crecimiento individual y colectivo.

Al respecto, Zeichner y Liston (1987) plantearon una serie de orientaciones, necesarias para el proceso formativo de los docentes: Competencia técnica y manejo de la clase atendiendo a habilidades y métodos adecuados; habilidades para analizar la práctica y realizar elecciones; conocimiento de la clase como una actividad con consecuencias éticas y morales y, sensibilidad hacia las necesidades de los alumnos. Todo ello, para tener un conocimiento real del contexto y de los procesos de su trabajo, participando en la creación de una política educativa, 
necesaria para tomar decisiones que afectan al aula, al centro y, en definitiva, a la comunidad.

Las aportaciones de Dalin (1983), McMahon et al. (1984), Hopkins (1988) esbozan una muestra de procesos, métodos y técnicas que tratan de facilitar el desarrollo colaborativo en los centros escolares. En este artículo presentamos una serie de fases susceptibles de variación dependiendo de unos u otros autores. Ninguna de las fases es rigurosamente antecedente o consecuente respeto a las demás, ya que el proceso puede iniciarse en cualquier punto. Algunas etapas o fases hacen referencia a procesos que han de iniciarse, mantenerse o ir logrando progresivamente a lo largo del tiempo. Las desarrollamos a continuación (Escudero, 1992, pp. 269 ss).

- Creación de una relación inicial para la mejora escolar. Todo proyecto de desarrollo educativo debe preparar las condiciones adecuadas para que el centro decida si implicarse o no. Requiere un proceso de diálogo, de clarificación y de compromiso así como la creación de espacios y tiempos adecuados.

- Creación de capacidades para la colaboración. Se trata de movilizar todos los recursos personales del centro, para apoyar, facilitar y mantener relaciones grupales que requieran del aprendizaje de capacidades y procedimientos grupales.

- Análisis y revisión de la escuela. Constituye un punto importante en el proceso colaborativo, centrado en la reflexión y discusión y en el análisis de los aspectos que necesitan mejorarse. Con todo ello, se pretende hacer un alto en el camino para tener una visión compartida de dónde estamos y hacia dónde deseamos ir.

- Identificación de ámbitos de mejora. A través de esta fase se clarifican y categorizan las situaciones problemáticas, priorizándolas de acuerdo con aquellos criterios que el grupo de profesores ha reconocido. Es un proceso necesario para profundizar en la comprensión conjunta de la propia realidad, debatiendo qué debería mejorarse a corto, medio y largo plazo.

- Clarificación y formulación de problemas. Cuando el grupo de profesores ha determinado ciertos ámbitos de mejora, será necesario emprender una serie de actividades que permitan su clasificación. Por ejemplo, determinar cómo y en qué se viene manifestando, cuáles son sus componentes, causas y consecuencias.

- Búsqueda de soluciones y preparación para la elaboración de planes de acción pedagógica. La mejora escolar requiere de un proceso complejo que permite ir buscando soluciones o alternativas de las que se derivan sus consecuencias. Algunas pueden relacionarse con la identificación o movilización de recursos y otras pueden requerir la presencia de agentes externos. Entre los procesos, característicos a esta fase, citamos: compartir materiales y experiencias de trabajo, la observación mutua, etc.

- Planificación. Cuando el grupo de profesores desea llevar a la práctica el conjunto de soluciones que se han identificado, se elabora un plan educativo conjunto. El grado de amplitud y generalidad de dicho plan dependerá de la naturaleza del ámbito de mejora sobre el que se está trabajando. La estructura de la planificación puede ser flexible, aunque debe suponer un esbozo de acción claro e ilustrativo para el aula y el centro. 
- Desarrollo del plan. Constituye una fase crucial en todo el proceso. Su puesta en práctica deriva del desarrollo crítico, reflexivo e investigador de las etapas anteriores. La relación colaborativa se considera decisiva como espacio de contraste, de aprendizaje sucesivo, de solución de problemas, de comprensión de lo que va ocurriendo y de elaboración de alternativas de mejora.

- La evaluación. El desarrollo colaborativo es un proceso evaluativo constante, orientado a la mejora educativa (Holly y Southworth, 1989). La evaluación subyace a todas las fases porque responde a criterios de revisión, análisis, juicio y toma de decisiones permanente. Mediante la evaluación se reflexiona colectivamente sobre los procesos conseguidos o no, estableciendo la continuidad entre lo realizado en un curso y el trabajo del año siguiente. El docente se considera un agente moral que evalúa su práctica desde criterior morales, reconociendo la problematicidad de los contextos educativos.

\section{CONCLUSIONES}

Enraizada en los presupuestos de la Escuela Nueva, Activa o Moderna, según los distintos autores que la construyeron, los centros educativos deben responder por un lado, a las necesidades de los alumnos teniendo en cuenta sus capacidades y por otro, al entorno social del que forman parte. En esta tarea, los docentes ocupan un lugar importante, necesitado de atención y apoyo profesional.

Frente a posiciones exclusivamente técnicas y reproductoras de la educación que priorizan la relación hegemónica de la teoría sobre la práctica, apostamos por una forma constructiva que enriquezca los contextos educativos, llenándolos de significado desde la reflexión en la acción. La teoría adquiere un papel relevante al fundamentar y justificar el conjunto de acciones e intervenciones del profesor que, a su vez, transforma y renueva los supuestos teóricos. Esta reconstrucción de la teoría y práctica educativa aboga por un profesional autónomo, crítico y reflexivo, capaz de propiciar el diálogo y el análisis, necesario en la resolución de sus problemas.

Para lograr este reto educativo, debemos atender con preferencia al período de prácticas de enseñanza de los futuros docentes desde dos ámbitos, la Universidad y el colegio. Este representa un momento clave en el crecimiento personal y profesional, que trata de conectar coherentemente los conocimientos estudiados en la Universidad y la dimensión cotidiana y rutinaria del aula y del centro. En algunos momentos, esta conexión resulta problemática para el alumno en prácticas como así lo reflejan las numerosas respuestas o comentarios que realizan y que en este artículo hemos presentado.

La literatura desarrollada al respecto esboza dos líneas de estudio: el conocimiento de la clase, necesario para la toma de decisiones y la creación de una estructura organizativa que permita al profesorado descubrir, analizar y solucionar conjuntamente, y en la medida de lo posible, los distintos conflictos que diariamente observan en sus aulas. Esta capacidad colaborativa afianza las relaciones entre los docentes y entre éstos y la Universidad. 
Desde la teoría y los programas de formación del profesorado debemos incidir en el desarrollo de habilidades socio-cognitivas que faciliten la reflexión, la toma de decisiones y el debate en los futuros docentes, atendiendo especialmente al período de prácticas de enseñanza.

\section{BIBLIOGRAFÍA}

DALIN, P. (1983): Can school learn? NFER, London.

DOYLE, W. (1977): The uses of nonverbal behavior: Toward an ecological model of classrooms. Merril-Palmer Quarterly. Vol. 23, pp. 179-192.

Doyle, W. (1979): Classroom effects. Teory into Practice. Vol. 18, $\mathrm{n}^{\circ} 3$, pp. 138-144.

Escudero, J. (1993): El centro como lugar de cambio educativo: La perspectiva de la colaboración. En GaIRIN, J. y ANTÚNEZ, S. (Ed): Organización escolar. Nuevas aportaciones. PPU, Barcelona, pp. 227-286.

Fultan, M. (1990): Staff development, innovation and institutional development. En JoYCE, B. (Ed.): Changing school culture through staff development. ASCD, Alexandría.

Gimeno, J. y FernánDez, M. (1980): La formación del profesorado de EGB. Análisis de la situación española. Ministerio de Universidades e Investigación, Madrid.

Holly, P. y SOUTHWORTH, G. (1989): The developing school. The Palmer Press, London.

HopkINS, D. (1988): Doing school based review, Instruments and guidelines. ACCO, Lovaina. IMBERNON, F. (1994): La formación del profesorado. Paidós, Barcelona.

Kemmis, S. (1988): El curriculum. Más allá de la teoría de la reproducción. Morata. Madrid.

KounIN, J. (1970): Disscipline and grouy management in classrooms. Holt, Rinehart and Winston, New York.

Kounin, J. y DoyLE, W. (1975): Degree of continuity of a lesson's signal system and the task involvement of children. Journal of Educational Psycbology. Vol. 67, pp. 159-164.

LAMPERT, M. (1985): How do teacher manage to teach? Perspectives on problems in practice. Harvard Educational Review. Vol. 5, no 19, pp. 1178-194.

LIEBERMAN, A. (1986): Building professional cultures in schools. Teacher College Press, New York.

MCMAHOM, A. (1984): Guidelines for revierw and internal development in school. School Council Programa. London.

SHERMAN, T. y CORMIER, W. (1974): An investigation of the influence of student behavior on teacher behavior. Journal of Applied Behavior Analysis, Vol. 7, pp. 1-30.

STHENHOUSE, L. (1984): Investigación y desarrollo del curriculum. Morata, Madrid.

TABACHNICK, T. (1980): Teacher education and the professional perspectives of student teachers. Interchange. Vol. 10, no 4, pp. 12-29.

Woods, P. (1977): Teaching for survival. En Woods, P. y Hammersley, M. (Eds.): School Experience. Martin's Press, New York.

ZEICHNER, K. (1992): Rethinking the practicum in the professional development school partnership. Journal of Teacher Education. № 4, pp. 296-307.

ZEICHNER, K. Y LISTON, D. (1987): Teaching student teachers to reflect. Harvard Educational Review. Vol. $57, \mathrm{n}^{\circ} 1$, pp. 23-47. 